\title{
Evaluación de la incorporación de residuos de cosecha sobre la producción orgánica de albahaca (Ocimum basilicum L.) en el valle de Mala
}

\author{
Evaluation of harvest residue incorporation on the organic basil (Ocimum basilicum L.) production \\ in the Mala valley
}

\author{
Saray Siura ${ }^{1}$; Ximena Reynafarje ${ }^{2}$; Félix Avila ${ }^{3}$
}

\section{Resumen}

En el valle de Mala, a $80 \mathrm{~km}$ al sur de Lima, se evaluó el efecto sobre el rendimiento y otras características agronómicas en cuatro cultivares de albahaca (Ocimum basilicum L.) de un sistema de producción orgánica, al incorporar al suelo residuos de cosecha de frijol (Phaseolus vulgaris L.). Se utilizó un diseño experimental de parcelas divididas, con los factores de incorporación de residuos como parcela principal y los cultivares (Della Riviera Ligure, Italian Large Leaf, Genovese y Rosie) como sub parcelas. La incorporación de residuos de frijol al suelo $\left(5,5 \mathrm{t} \mathrm{ha}^{-1}\right)$ incrementó el rendimiento $\left(21,8 \mathrm{t} \times \mathrm{ha}^{-1}\right)$, la altura de planta, el área foliar y el peso seco de hojas con diferencias significativas. En cuanto a los cultivares, se encontraron diferencias altamente significativas para el rendimiento, altura de planta y área foliar; el mayor rendimiento se obtuvo con el cultivar Della Riviera Ligure $\left(23,5 \mathrm{t} \times \mathrm{ha}^{-1}\right)$. No se encontró diferencias estadísticas significativas en la interacción de ambos factores. Se concluye que la incorporación de residuos de frijol antes de la siembra incrementa el rendimiento y que los cultivares de albahaca verde obtuvieron un mayor rendimiento que el cultivar rojo Rosie. Los altos rendimientos obtenidos en este ensayo permiten mostrar el potencial productivo del cultivo de albahaca en un sistema de producción orgánica.

Palabras clave: albahaca; incorporación de residuos; Ocimum; producción orgánica; rendimiento en fresco.

\begin{abstract}
At Mala valley, located $80 \mathrm{~km}$ from Lima-Peru, a field experiment was conducted to evaluate the effects of bean (Phaseolus vulgaris L.) residue incorporation on yield and other agronomical characteristics of four basil (Ocimum baislicum L.) cultivars in an organic production system. A split plot experimental design was used with the residues incorporation factors as main plot and cultivars (Della Riviera Ligure, Italian Large Leaf, Genovese y Rosie) as sub plots. Bean residue biomass incorporation $\left(5,5 \mathrm{t} \times \mathrm{ha}^{-1}\right)$ resulted on a higher yield $\left(21,8 \mathrm{t} \times \mathrm{ha}^{-1}\right)$, dry weight, plant height and foliar area with significant differences. In terms of the cultivars high significant differences were found for yield, plant height and foliar area; the highest yield was for cultivar Della Riviera Ligure $\left(23,5 \mathrm{t} \times \mathrm{ha}^{-1}\right)$. No statistically significant differences were found in the interaction of both factors. It's concluded that bean residue incorporation before planting increases yield. Green basil cultivars reached a higher yield than red cultivar Rosie. The high yields obtained in this study allow us to show the productive potential of basil crop in organic farming system.
\end{abstract}

Keywords: basil; residue incorporation; Ocimum; organic production; fresh market yield.

\section{Introducción}

La demanda de productos orgánicos a nivel mundial sigue creciendo debido, entre muchas razones, a la preocupación de los consumidores por la relación entre alimentación y salud, pero también por los beneficios que generan para el medio ambiente (Lester, 2006). El Perú no es ajeno a esta situación y, en los últimos años, impulsados por el auge de la gastronomía local y exportación, se vienen generando nuevos mercados para estos productos, especialmente hierbas aromáticas y culinarias, como la albahaca. El mercado local de productos orgánicos llega a generar entre 13,1 y 23,2 millones de dólares americanos por año (Martínez, 2012).
Los consumidores de lo orgánico generan oportunidades para los pequeños productores de hortalizas orgánicas y, si tomamos en cuenta que el $85 \%$ de los agricultores en el Perú tienen parcelas con menos de 10 ha (MINAG, 2009), resulta importante generar información sobre prácticas ecológicas de cultivo e introducción de nuevos cultivares en sistemas de producción orgánica, que permitan incrementar el rendimiento y la calidad de los productos cosechados.

Una de estas prácticas es la incorporación de residuos de cultivo que se utilizan en el manejo de hortalizas para mejorar la fertilidad del suelo. Entre estos encontramos algunas fabáceas comestibles como el frijol, la arveja y el

\footnotetext{
Universidad Nacional Agraria La Molina, Lima, Perú. Email: saray@lamolina.edu.pe

Universidad Nacional Agraria La Molina, Lima, Perú. Email: huerto@lamolina.edu.pe

Asociación de Productores Ecológicos Biofrut.
} 
haba que se mencionan como los más importantes para ser incorporados antes de la siembra del siguiente cultivo y se constituyen, además, en una alternativa de rotación o de asociación con otro cultivo (Ulloa et al., 2011; Rivero, 2006; Gliessman, 2002; Barreto et al.,1994; Guerrero, 1993).

La albahaca es una hortaliza muy apreciada tanto para su consumo en fresco como procesado, pero también por su contenido de aceites esenciales utilizados como aromatizantes y saborizantes en la industria de alimentos, la farmacéutica y la cosmética, así como por sus propiedades antimicrobianas e insecticidas (Bozin et al., 2006; Suppakul et al., 2003; Aslan et al., 2004; Bowers y Nishida, 1980). La albahaca en fresco ha experimentado una mayor demanda por la versatilidad de su uso, asociado a propiedades nutraceúticas y a su demanda en los mercados gourmet, sobre todo si se trata de albahaca orgánica (Succop y Newman, 2004).

El objetivo de este trabajo es evaluar el efecto de la incorporación de residuos de frijol antes de la siembra sobre el rendimiento y otras características agronómicas de cuatro cultivares de albahaca $(O$. basilicum L.) en un sistema de producción orgánica, manejado por pequeños agricultores del valle de Mala.

\section{Materiales y métodos}

El experimento se llevó a cabo entre los meses de febrero y agosto de 2011, en una parcela comercial de albahaca, de la Asociación BIOFRUT, organización de pequeños productores orgánicos del valle de Mala. La ubicación geográfica de la parcela es de $12^{\circ} 33^{\prime} 17^{\prime}$ 'S, $76^{\circ} 35^{\prime} 36^{\prime \prime O}$ y una altitud de $200 \mathrm{msnm}$. La temperatura promedio durante el ensayo fue de $18,6^{\circ} \mathrm{C}$, con una máxima de $25,2^{\circ} \mathrm{C}$ y una mínima de $16,1^{\circ} \mathrm{C}$; la humedad relativa fluctuó entre $79 \%$ y $94 \%$, siendo ambos factores favorables para el cultivo. En cuanto a la semilla, se seleccionaron los cultivares de albahaca más utilizados para el mercado fresco y un nuevo cultivar de albahaca roja, seleccionado por su potencial para el mercado fresco gourmet. Las semillas empleadas fueron: Della Riviera Ligure (DRL, verde oscuro); Genovese (G, verde); Italian Large Leaf (ILL, verde de hojas extra grande) y Rosie (R, roja de hojas pequeñas). Los residuos de cosecha incorporados correspondieron a una siembra de frijol blanco Tipo I, picados e incorporados mediante tracción mecánica, treinta días antes de la siembra de albahaca. El total de biomasa incorporada fue de 5,5 $\mathrm{t} \times \mathrm{ha}^{-1} \mathrm{y}$ se determinó al final de la cosecha de frijol tomando una muestra de 10 plantas por cada $200 \mathrm{~m}^{2}$, las cuales fueron secadas en estufa a $60{ }^{\circ} \mathrm{C}$, alcanzando un porcentaje promedio de $21 \%$ de materia seca.

El suelo utilizado en el ensayo fue de textura franca (Tabla 1 ), en el que se hicieron aplicaciones de estiércol de vacuno $\left(20 \mathrm{t} \times \mathrm{ha}^{-1}\right)$ durante la preparación del terreno y compost $\left(10 \mathrm{t} \times \mathrm{ha}^{-1}\right)$ en toda la parcela del agricultor, de acuerdo con las prácticas seguidas por el agricultor antes de la siembra. La siembra se realizó por trasplante, con plantas de 30 días de almacigado, con una población total de 81.000 plantas/ ha. El riego fue por gravedad, cada 5 a 15 días, tomando en cuenta la humedad del suelo y la disponibilidad de agua. En la Tabla 2, se muestran los resultados del análisis del agua de riego, donde se observa que no fue un factor limitante para el suelo ni para el cultivo.

El manejo de plagas y enfermedades se hizo de manera preventiva, instalando trampas pegantes de plástico de color amarillo para adultos de mosca minadora (Lyriomiza huidobrensis) y mosca blanca (Bemisia tabaci) $y$ realizando monitoreos continuos. Se hicieron aplicaciones de productos permitidos para producción orgánica como extracto de cítricos (DESFAN) y espolvoreos de azufre para el mildiu (Peronospora destructor). Se presentaron daños aislados de comedor de hoja o gusano medidor (Pseudoplusia includens), pero sin llegar a umbrales económicos.

La primera cosecha se realizó 30 días después del trasplante con plantas de 25 a $35 \mathrm{~cm}$ de altura. Los cortes se realizaron antes de la floración de acuerdo con las exigencias del mercado fresco, con una frecuencia de 15 días durante cuatro meses, alcanzando un total de siete cortes.

Los tratamientos fueron determinados en base a la combinación de los factores siguientes: incorporación de residuos y cultivares de albahaca, dando un total de ocho tratamientos (Tabla 3). El diseño estadístico utilizado fue de Parcelas Divididas, donde el factor incorporación de residuos fue asignado a las parcelas principales, con un diseño de bloques completamente al azar (DBCA), con cuatro repeticiones y el factor cultivar a las subparcelas. Se utilizó la prueba de TUKEY (alpha=0,05) para comparar las medias de los tratamientos. Los cálculos

Tabla 1. Características del suelo utilizado en el ensayo de albahaca. Mala 2011

\begin{tabular}{|c|c|c|c|c|c|c|c|c|c|c|c|c|c|c|c|}
\hline $\begin{array}{c}\mathrm{pH} \\
(1: 1) \\
\end{array}$ & $\begin{array}{c}\text { Arena } \\
(\%)\end{array}$ & $\begin{array}{c}\text { Limo } \\
(\%)\end{array}$ & $\begin{array}{c}\text { Arcilla } \\
(\%)\end{array}$ & $\begin{array}{c}\text { Clase } \\
\text { Textural } \\
\end{array}$ & $\begin{array}{l}\text { C.E. } \\
(1: 1) \\
\text { dS/m }\end{array}$ & $\begin{array}{c}\mathrm{CaCO}_{3} \\
\% \\
\end{array}$ & $\begin{array}{c}\text { M.O. } \\
\%\end{array}$ & $\begin{array}{c}\mathbf{P} \\
\text { (p.p.m) }\end{array}$ & $\begin{array}{c}\mathrm{K} \\
\text { (p.p.m) }\end{array}$ & $\begin{array}{c}\text { C.I.C. } \\
\text { (meq/100g) }\end{array}$ & $\mathrm{Ca}^{+2}$ & $\mathbf{M g}^{+2}$ & $\mathbf{K}^{+}$ & $\mathbf{N a}^{+}$ & $\begin{array}{l}\mathbf{A l}^{+3} \\
+\mathbf{H}^{+} \\
\end{array}$ \\
\hline 7,6 & 42 & 38 & 20 & franco & 0,67 & 1,3 & 2,8 & 40,2 & 246 & 12,32 & 9,5 & 2,05 & 0,60 & 0,2 & 0 \\
\hline
\end{tabular}

Fuente: UNALM, Laboratorio de suelos, plantas, agua y fertilizantes, 2011.

Tabla 2. Análisis de agua de riego utilizada en el ensayo de albahaca. Mala 2011

\begin{tabular}{|c|c|c|c|c|c|c|c|c|c|c|c|c|c|c|c|c|}
\hline pH & $\begin{array}{l}\text { C.E. } \\
\text { (dS/m) }\end{array}$ & $\begin{array}{c}\text { Calcio } \\
(\mathrm{meq} / \mathrm{L})\end{array}$ & $\underset{(\mathrm{meq} / \mathrm{L})}{\mathbf{M g}}$ & $\begin{array}{l}\text { Potasio } \\
(\mathrm{meq} / \mathrm{L})\end{array}$ & $\begin{array}{c}\text { Sodio } \\
\text { (meq/L) }\end{array}$ & $\begin{array}{l}\text { suma de } \\
\text { cationes }\end{array}$ & $\begin{array}{l}\text { Nitratos } \\
(\mathrm{meq} / \mathrm{L})\end{array}$ & $\begin{array}{c}\text { Carbonatos } \\
(\text { meq/L) }\end{array}$ & $\begin{array}{c}\text { Bicarbonatos } \\
\text { (meq/L) }\end{array}$ & $\begin{array}{l}\text { Sulfatos } \\
\text { (meq/L) }\end{array}$ & $\begin{array}{c}\text { Cloruros } \\
\text { (meq/L) }\end{array}$ & $\begin{array}{c}\text { Suma } \\
\text { de } \\
\text { aniones }\end{array}$ & $\begin{array}{c}\text { Sodio } \\
(\%)\end{array}$ & RAS & $\begin{array}{c}\text { Boro } \\
(\mathrm{ppm})\end{array}$ & Clasif. \\
\hline 7,68 & 0,12 & 0,66 & 0,2 & 0,03 & 0,4 & 1,29 & 0,01 & 0 & 0,98 & 0,04 & 0,3 & 1,33 & 31,01 & 0,61 & 0,05 & C1-S1 \\
\hline
\end{tabular}

Fuente: UNALM, Laboratorio de suelos, plantas, agua y fertilizantes, 2011. 
estadísticos se realizaron utilizando el software estadístico SAS (Statistical Analysis System).

Las evaluaciones realizadas fueron: altura de planta (muestra semanal de cinco plantas por sub-parcela hasta la primera cosecha); peso fresco (biomasa total cosechada); peso seco (muestra fresca secada en estufa a $60{ }^{\circ} \mathrm{C}$ ) y área foliar (muestra de cinco plantas 60 días después del trasplante), de acuerdo con el método de la silueta de papel (Martínez, 2011).

\section{Resultados y discusión Altura de planta}

Una mayor altura de planta en el cultivo de albahaca está relacionada con la calidad del producto, aunque depende de factores como el cultivar, temperatura, densidad de plantas, riego y abono, entre otros. En el campo, la altura

Tabla 3. Tratamientos utilizados en el ensayo

\begin{tabular}{lll}
\hline & $\begin{array}{c}\text { Incorporación de } \\
\text { residuos }\end{array}$ & \multicolumn{1}{c}{ Cultivar } \\
T1 & & Rosie \\
T2 & Con incorporación & Italian Large Leaf \\
T3 & & Della Riviera Ligure \\
T4 & & Genovese \\
\hline T5 & & Rosie \\
T6 & Sin incorporación & Italian Large Leaf \\
T7 & & Della Riviera Ligure \\
T8 & & Genovese \\
\hline
\end{tabular}

de planta antes de la floración se asocia con mayor vigor, volumen y calidad. En el mercado local, la mayor altura facilita la manipulación y el corte progresivo de tallos durante la comercialización, alargando la vida del producto cosechado.

En este ensayo, la incorporación de residuos de frijol influyó sobre la altura de planta, con diferencias significativas y altamente significativas entre los cultivares (Tabla 4); los cultivares de hoja verde (DRL, ILL y G) presentaron una mayor altura que el cultivar de rojo Rosie, que es un cultivar de porte bajo.

La interacción de los factores en estudio no fue estadísticamente significativa, mostrando que cada uno de estos tuvo un efecto independiente sobre la altura de la planta.

La incorporación de residuos de frijol, al ser una característica de calidad del producto, puede ser incorporada como una práctica que influye positivamente, como también los cultivares.

\section{Área foliar}

El área foliar es un indicador de la eficiencia fisiológica de la planta, relacionado en forma directa con la cantidad de clorofila y la capacidad de fotosíntesis que se expresa en la ganancia de materia seca, permitiendo medir el crecimiento y desarrollo de la planta (Cardona, Aramendiz y Barrera, 2006); en cultivos de hoja como la albahaca, esta variable también ayuda a definir la época de siembra óptima relacionándola con factores como temperatura y radiación.

En la producción y comercialización para mercado fresco, un mayor tamaño y número de hojas inciden en una

Tabla 4. Altura de planta, área foliar, rendimiento en fresco y porcentaje de materia de seca de cuatro cultivares de albahaca $(O$. basilicum $\mathrm{L}$. ) sembrados en suelo con y sin incorporación de residuos de frijol

\begin{tabular}{lcccc}
\hline & $\begin{array}{c}\text { Altura de planta } \\
(\mathbf{c m}) \\
\mathbf{3 6} \mathbf{~ D D T}\end{array}$ & $\begin{array}{c}\text { Área foliar } \\
\left(\mathbf{c m}^{\mathbf{2}}\right)\end{array}$ & $\begin{array}{c}\text { Rendimiento } \\
\text { en fresco } \\
\left(\mathbf{t}^{*} \mathbf{h a}^{-\mathbf{1}}\right)\end{array}$ & $\begin{array}{c}\text { Materia seca de } \\
\text { hojas (\%) }\end{array}$ \\
\hline INCORPORACIÓN (IN) & & & & \\
$\quad$ Con incorporación (I) & $30,4 \mathrm{a}$ & $1867 \mathrm{a}$ & $21,8 \mathrm{a}$ & $23,8 \mathrm{a}$ \\
$\quad$ Sin incorporación (NI) & $27,6 \mathrm{~b}$ & $1812 \mathrm{~b}$ & $17,4 \mathrm{~b}$ & $17,2 \mathrm{~b}$ \\
CULTIVAR (CV) & & & & \\
Rosie & $22 \mathrm{~b}$ & $1737 \mathrm{~b}$ & $11,2 \mathrm{~b}$ & $21,3 \mathrm{a}$ \\
Italian Large Leaf & $35 \mathrm{a}$ & $1863 \mathrm{a}$ & $22,2 \mathrm{a}$ & $19,5 \mathrm{a}$ \\
Della Riviera Ligure & $28 \mathrm{a}$ & $1906 \mathrm{a}$ & $23,5 \mathrm{a}$ & $20,5 \mathrm{a}$ \\
Genovese & $29 \mathrm{a}$ & $1851 \mathrm{~b}$ & $21,3 \mathrm{a}$ & $20,6 \mathrm{a}$ \\
ANOVA & & & & \\
INCORPORACIÓN & $*$ & $* *$ & $*$ & n.s. \\
CULTIVAR & $* *$ & $* *$ & $* *$ & n.s. \\
IN x CV & n.s. & n.s. & &
\end{tabular}

Significativo a $\mathrm{P}<0,05$.

** Significativo con $\mathrm{P}<0,01$. 
mayor cantidad de producto comestible y, por lo tanto, se considera como un indicador de calidad.

En el presente ensayo, se encontraron diferencias estadísticamente significativas para los factores de incorporación de residuos de fríjol y cultivar (Tabla 4). La integración de residuos influyó sobre una mayor área foliar, lo que en el mercado fresco se considera como un factor de calidad debido al mayor tamaño de planta o de hojas cosechadas, ya que la comercialización se realiza por paquetes (tallos agrupados en un volumen conocido).

Asímismo, los cultivares de hoja verde se vieron influenciados por la incorporación de residuos de frijol, siendo mayores al promedio alcanzado sin incorporación. El cultivar Rosie obtuvo los menores valores, debido a su menor tamaño de planta por tratarse de un producto delicado para el mercado gourmet. La interacción entre ambos factores no mostró diferencias significativas por lo que se considera que estos influyen de manera independiente sobre el área foliar. Las diferencias obtenidas entre los cultivares están relacionadas directamente con la morfología propia de cada cultivar.

Estos resultados coinciden con los obtenidos por Benito y Chiesa (2000) que estudiaron parámetros fisológicos en cultivares de albahaca y obtuvieron valores de área foliar que oscilaron entre los 980 y los $1.920 \mathrm{~cm}^{2}$.

\section{Rendimiento}

El rendimiento de albahaca se evaluó en fresco debido al mercado de destino del producto. En el Perú, el rendimiento promedio nacional es de $8,7 \mathrm{t} \times \mathrm{ha}^{-1}$ alcanzando los más altos valores en la región Arequipa con $12,1 \mathrm{t} \times \mathrm{ha}^{-1} \mathrm{y}$ los menores en la región Apurímac con 5,5 t× $\mathrm{ha}^{-1}$ (MINAG, 2009). No se encuentran fácilmente estadísticas de producción de albahaca en otros países, pero en Colombia, la Corporación Colombiana Internacional (CCI, 2011) reporta rendimientos en fresco entre 11,2 y $23,5 \mathrm{t} \times \mathrm{ha}^{-1}$. Con un rango más amplio de rendimiento en fresco $(11,2$ y 23,52 $\left.\mathrm{t} \times \mathrm{ha}^{-1}\right)$, los resultados de este ensayo demuestran el alto potencial del cultivo de albahaca en el valle de Mala. Se encontraron diferencias estadísticamente significativas en el caso de la incorporación de residuos de frijol, mientras que para los cultivares se encontraron diferencias altamente significativas. Sin embargo, la interacción de estos dos factores no fue estadísticamente significativa (Tabla 4), influyendo de manera independiente sobre el rendimiento en fresco de la albahaca. Cuando se incorporaron los residuos de frijol, el rendimiento fue superior hasta en un $26 \%$ al rendimiento total, comparado con el tratamiento donde no se hizo la incorporación. En cuanto a los cultivares, los de hoja verde fueron superiores, estadísticamente, al cultivar Rosie de hoja roja. El mayor rendimiento $\left(23,5 \mathrm{t} \times \mathrm{ha}^{-1}\right)$ se obtuvo con el cultivar Della Riviera Ligure (Tabla 4).

Los cultivares de hoja verde se destinan para el mercado en fresco o la extracción de aceites esenciales. En cuanto al mercado fresco, las características ligadas a mejor calidad y precio son la altura de planta, tamaño de hojas, volumen de planta. Para procesamiento, a estas características se añade el porcentaje de materia seca (de 18 a $25 \%$ ). En el caso de los cultivares rojos como Rosie, por tratarse de un nicho de mercado, ni el peso ni el volumen determinan la calidad sino más bien la intensidad del color, ya que se trata de un cultivar de porte más pequeño y menor biomasa, pero de mayor precio por ser un producto gourmet. Por estas razones y por los rendimientos alcanzados, la perspectiva del cultivo orgánico de albahaca verde y roja en el valle de Mala, se muestra muy interesante como una alternativa de rotación después del frijol, aprovechando los residuos para la mejora de la productividad del siguiente cultivo. Los rendimientos obtenidos en este ensayo se consideren altos comparados con los promedios nacionales y los obtenidos en otros experimentos con albahaca de 6,1 a 8,3 tha-1 (Ruiz et al., 2009) y de 15 a $21 \mathrm{t} \times \mathrm{ha}^{-1}$ (Palada, 2000).

\section{Porcentaje de materia seca en hojas}

La acumulación de materia seca es un factor que nos permite conocer la eficiencia biológica del cultivo y nos permite determinar el momento de máxima ganancia de carbohidratos antes de la floración, que es el momento de cosecha comercial de este cultivo. Se evaluó el porcentaje de materia seca de hojas obteniendo diferencias altamente significativas para el factor de incorporación de residuos de frijol (Tabla 4), mostrando que esta práctica de manejo agronómico puede ser directamente favorable para la producción de albahaca orgánica, tanto para el mercado fresco como para procesamiento $(23,76 \%)$, ya que mejora su calidad. Por otro lado los cultivares no mostraron diferencias estadísticas significativas para el porcentaje de materia seca, fluctuando entre $19,5 \%$ (ILL) y $21,32 \%$ (R) (Tabla 4). Estos resultados nos estarían demostrando que las diferencias en el rendimiento en fresco estarían más relacionadas con la mayor área foliar y el mayor contenido de humedad de las hojas de los cultivares verdes.

A pesar del menor rendimiento alcanzado, el cultivar Rosie obtuvo el mayor porcentaje de materia seca, aunque sin diferencias estadísticas significativas con los demás cultivares. Este mayor contenido de materia seca estaría relacionado también con una mayor acumulación de aceites esenciales.

\section{Conclusiones}

La incorporación de residuos de cosecha de frijol antes de la siembra incrementa el rendimiento, la altura de planta, el área foliar y el porcentaje de materia seca del cultivo de albahaca orgánica para mercado fresco, en el valle de Mala. Existe un gran potencial para el cultivo de albahaca orgánica en el valle de Mala, con rendimientos de hasta 21,8 $\mathrm{t} \times \mathrm{ha}^{-1}$ cuando se incorporan residuos de frijol antes de la siembra.

Los cultivares de albahaca alcanzaron rendimientos entre 11,2 $\mathrm{t} \times \mathrm{ha}^{-1}$ y $23,5 \mathrm{t} \times \mathrm{ha}^{-1}$. El cultivar Della Riviera Ligure obtuvo el mayor rendimiento mientras que el cultivar Rosie obtuvo el menor rendimien $\mathrm{t} \times \mathrm{ha}^{-1}$ ) en fresco.

Se encontraron diferencias entre cultivares de albahaca en cuanto a altura de planta, área foliar y porcentaje de materia seca de hojas. La incorporación de residuos de frijol antes 
de la siembra de albahaca constituye una alternativa para incrementar el rendimiento del cultivo de albahaca para mercado fresco.

Los resultados obtenidos en este ensayo son alentadores en cuanto a la viabilidad de la producción orgánica de hortalizas frescas como albahaca y la introducción de nuevos cultivares con un alto potencial de rendimiento (hasta 23,5 $\mathrm{t} \times \mathrm{ha}^{-1}$ ) comparados con el rendimiento promedio nacional $\left(8,1 \mathrm{t} \times \mathrm{ha}^{-1}\right)$.

\section{Literatura citada}

Aslan, I.; Ozbek, H.; Calmasur, O. y Sahin, F. 2004. Toxicity of essential oil vapors to two greenhouse pests, Tetranychus urticae Koch and Bemisia Tabaci Genn. 19: 167-173.

Barreto, H.J.; Pérez, C.; Fuentes, M.; Queme, J.L. y Larios, L. 1994. Efecto de la dosis de UREA-N en el rendimiento del maíz en un sistema de rotación con leguminosas de cobertura. Agronomía Mesoamericana, 5, 88-95.

Benito, A. y Chiesa, A. 2000. Parámetros fisiológicos y productivos en cultivares de albahaca Ocimum basilicum L. Revista FAVE, 14(1):19-28.

Bowers, W.S. y Nishida, R. 1980. Juvocimenes: Potent juvenile hormones mimics from sweet basil. Science, 209: 1030-1032.

Bozin, B.; Mimica-Dukic, N.; Simin, N. y Anackov, G. 2006. Characterization of the volatile composition of essential oils of some Lamiaceae species and the antimicrobial and antioxidant activities of the entire oils. $J$. Agr. Food Chem, 54: 1822-1828.

Cardona, C.; Aramendiz, H. y Barrera, C. 2006. Modelo para estimación de área foliar en berenjena (Solanum melongena L) basado en modelo no destructivo. Revista de la Universidad Nacional de Córdoba. Departamento de Ingeniería Agronómica y Desarrollo Rural.

Corporación Colombiana Internacional [CCI]. 2011. Plan Hortícola Nacional. Recuperado de http://www.cci. org.co/publicaciones/1_PHNfinal.pdf

Gliessman, S. 2002. Agroecología: Procesos Ecológicos en la Agricultura Sostenible. Ed. CATIE.

Guerrero, J. 1993. Abonos Orgánicos: tecnología para el Manejo Ecológico de Suelos. Lima, Perú: Ed. RAAA.

Lester, G. 2006. Organic versus conventionally grown produce: quality differences and guidelines for comparison studies. Hort. Science, 41(2): 296-300.

Martínez, G. 2011. Medidas de crecimiento y su dinámica en organismos multicelulares recopilación de fórmulas $e$ indices aplicados en biología y fisiología vegetal. Disponible en http://es.scribd.com/doc/62280900/ Medidas-de-crecimiento.

Martinez, J. 2012. Perú: The market for organic products. FiBL-IFOAM Report. Bonn, Germany: Research institute of organic agriculture (FiBL), Frick, Switzerland and International Federation for Organic Agriculture Movement (IFOAM).
Ministerio de Agricultura [MINAG]. 2009. Estadística Agraria mensual. Disponible en http://www.minag.gob.pe/ boletines/estadistica-agraria-mensual.html

Palada, M.; Crossman, S.; Kowalski, J. y Collingwood, C. 2000. Evaluation of Organic and Synthetic Mulches for Basil Production under Drip Irrigation. Journal of Herbs, Spices \& Medicinal Plants, 6 (4): 39-48.

Rivero, C.; Torres, A. y Ampuero, J. 2006. Efecto del uso de residuos orgánicos y fertilización sobre el comportamiento del nitrógeno mineral en el suelo. Revista Facultad de Agronomía, 32:1-13.

Ruiz, F.; Vásquez, C.; García, J. y Salazar, E. 2009. Comparación del Costo energético de dos manejos de suelo para Albahaca. TERRA Latinoamericana, 27(4):383-389.

Succop, E. y Newman, S. 2004. Organic fertilization of fresh market sweet basil in a greenhouse. Hort. Technology, 14: 243-250.

Suppakul, P.; Miltz, J.; Sonneveld, K. y Bigger, S.W. 2003. Antimicrobial properties of basil and its possible application in food packaging. J. Agr. Food Chem, 51: 3197-3207.

Ulloa, J.A.; Rosas, P.; Ramirez, J.C. y Ulloa, B.E.2011. El frijol (Phaseolus vulgaris): su importancia nutricional y como fuente de fitoquímicos. Revista Fuente, Año 3(8). Universidad Autónoma de Nayarit. Nayarit, México. 